\title{
VISIBLE LIGHT EMISSION FROM POROUS SILICON
}

\author{
M. Bugajski, M. Wesolowski, W. Lewandowski, J. Ornoch \\ AND J. KĄTCKI
}

Institute of Electron Technology, Al. Lotników 32/46, 02-668 Warszawa, Poland

The aim of this paper is the study of porous $\mathrm{Si}$ prepared by preferential anodic dissolution in concentrated HF acid solutions. Porous silicon layers exhibited extremely efficient luminescence in the $700-900 \mathrm{~nm}$ range at room temperature. Basic characteristics of this luminescence strongly suggest the intrinsic origin of the process, directly related to quantum confinement. The additional transmission-electron-microscopy and electron-diffraction studies. were performed to support hypothesis that luminescence originates from silicon nanostructures.

PACS numbers: 78.65.-s

\section{Introduction}

Porous silicon formation may occur during anodic polarization in concentrated HF solutions [1]. The material was considered as an isolation dielectric in integrated circuits some time ago [2]. Recently, photoluminescence in the near-infrared [3] and later intense luminescence in the visible range of the spectrum [4] have been reported for porous silicon. The discovery that porous silicon can emit visible light at room temperature has once again generated a worldwide interest in this material. Most of the current research on light emission in porous silicon has been directed at understanding the origin of this luminescence which is crucial for further development of the material. "Blue shift" of the emission from porous material, comparing to bulk silicon, has been originally attributed to quantum size effects in a network of free standing wires composing porous layer [3], although dihydride passivation $\left(\mathrm{SiH}_{2}\right)$ [5] and near surface amorphous films [6] have also been found important in light generation process. There are also claims that luminescence in porous silicon is not an intrinsic material property but is due to molecules chemisorbed to the vast surface area provided by porous material [7] or even originates from the presence of siloxene $\left(\mathrm{Si}_{6} \mathrm{O}_{3} \mathrm{H}_{6}\right)$ and its derivatives formed in porous Si during etching process [8].

In this paper we describe the results of luminescence and transmission-electron-microscopy (TEM) studies of porous Si formed by anodic dissolution in HF based electrolytes. 


\section{Experimental}

Porous silicon layers were grown on polished surface of single crystalline silicon wafers in $\mathrm{HF}(48 \mathrm{wt} \%)-\mathrm{C}_{2} \mathrm{H}_{5} \mathrm{OH}$ (98 wt\%) solution (1:1 to $5: 1$ by volume) under conditions of constant current. A special teflon cell was constructed providing good quality electrical contact to the back side of the sample, resulting in a uniform growth of porous layer. The cell assembly was immersed in electrolyte and a constant voltage was applied between the sample and $\mathrm{Pt}$ counterelectrode (cathode). Depending on the sample resistivity a different voltage had to be applied to force a given current density across the sample. After etching the wafers were rinsed with deionized water and dried in air.

\section{Results}

Varying anodization conditions, i.e. concentration of the electrolyte current, density and anodization time, and using material of different resistivity, it was possible to obtain samples with different porosity exhibiting luminescence at room temperature in the 700-900 nm range. A typical photoluminescence (PL) spectrum of the sample anodized at current density $50 \mathrm{~mA} / \mathrm{cm}^{2}$, for $20 \mathrm{~min}$ in 1:1 (98 wt\% $\mathrm{C}_{2} \mathrm{H}_{5} \mathrm{OH}, 48 \mathrm{wt} \% \mathrm{HF}$ ) solution is shown in Fig. 1 . The luminescence is clearly visible by the naked eye (bright red color) and its quantum efficiency is comparable to the efficiency of the best AlGaAs material. The peak position of the PL band is weakly temperature dependent and both positive and negative temperature shifts in certain temperature ranges were observed for different samples. The band is symmetric and the total integrated PL intensity depends sublinearly on excitation intensity. Using higher HF concentrations in the electrolyte (5:1 instead of 1:1) lower porosity samples can be fabricated exhibiting luminescence with maximum at $850 \mathrm{~nm}-900 \mathrm{~nm}$. The shift of Pi maximum to shorter wavelengths (higher energies) with increasing porosity (i.e. decreasing width of the columns left by the etching process) is compatible with hypothesis that luminescence originates from quantum confinement of the carriers.

Porous silicon layer can be easily separated from the silicon substrate either by prolonged etching (internal strain in the porous layer exceeds critical value and causes the layer to lift off) or by reducing the electrolyte concentration and increasing current density above $100 \mathrm{~mA} / \mathrm{cm}^{2}$ (electropolishing regime). The porous silicon layer separated from the substrate breaks and curls up upon drying. Pore propagation rate determined from the thickness of porous layers was estimated for $15-20 \mathrm{~nm} / \mathrm{s}$.

Porous silicon layers separated from the substrate appear to be translucent, from dark red to orange depending on the porosity. Figure 2 shows cross-sectional TEM micrograph of a porous layer. The layer thickness is about $1 \mu \mathrm{m}$. The porous structure is characterized by randomly spaced parallel sided pores with a diameter of about $0.1 \mu \mathrm{m}$. We have not found any preferential crystallographic etching in porous silicon. Generally, pore propagation follows the current direction (particularly in heavily doped, low resistivity silicon). Figure 3 shows plane-view TEM micrograph of porous silicon layer separated from the substrate, for which no further specimen preparation is required. 


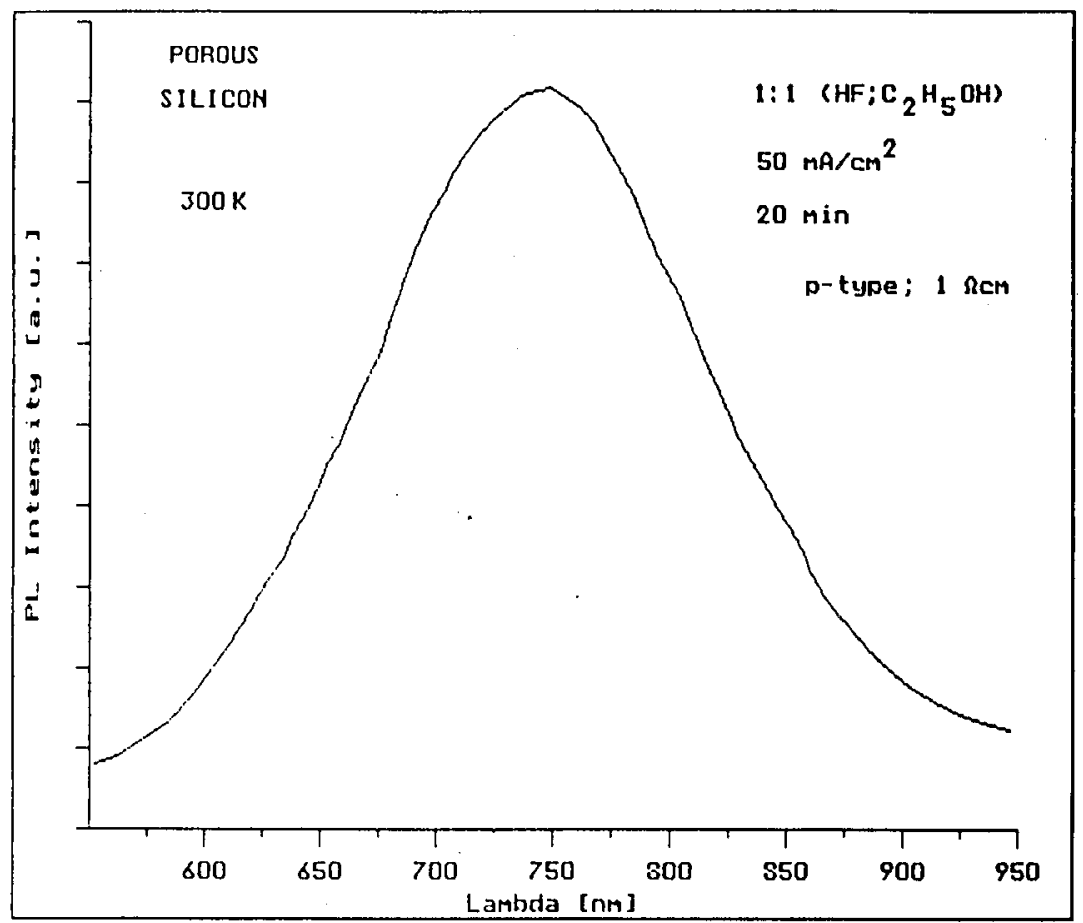

Fig. 1. Room temperature PL spectrum of porous silicon produced by anodic etching of $p$-type $1-5 \Omega \mathrm{cm}$ silicon for $20 \mathrm{~min}$ at $50 \mathrm{~mA} / \mathrm{cm}^{2}$.

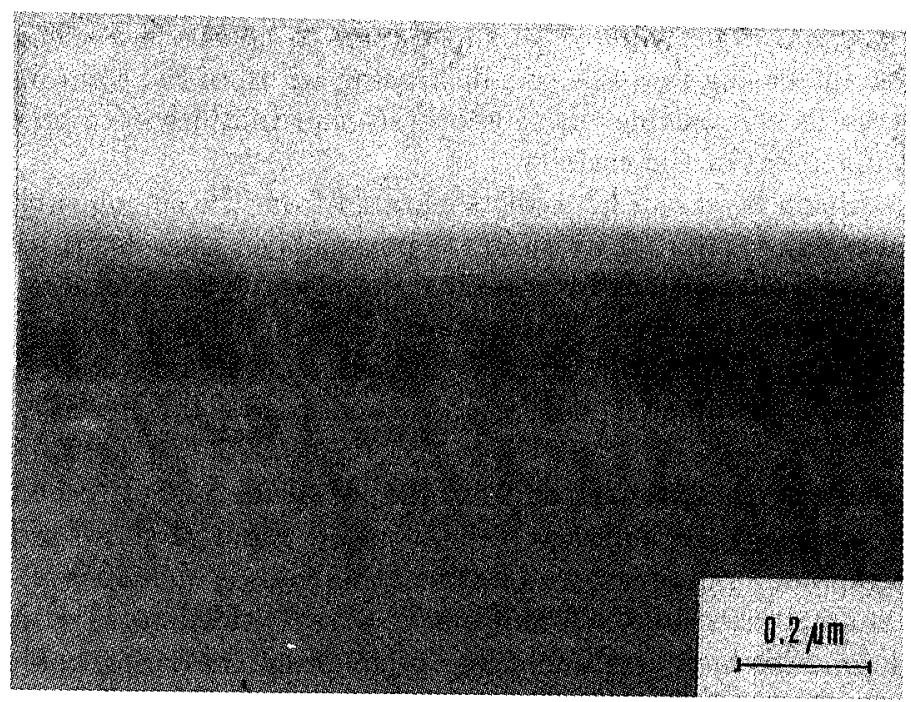

Fig. 2. Cross-sectional. TEM micrograph of a porous layer formed on 1-5 $\Omega \mathrm{cm} p$-type silicon at $50 \mathrm{~mA} / \mathrm{cm}^{2}$. 


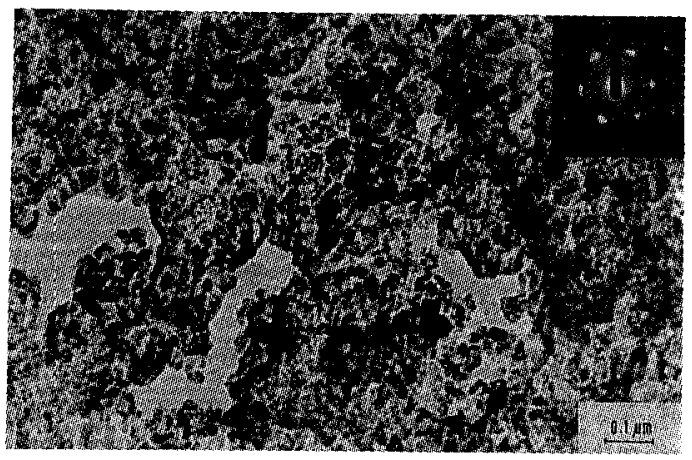

Fig. 3. Plane-view TEM micrograph of porous silicon layer separated from the substrate. Porous layer has been formed on p-type 1-5 $\Omega \mathrm{cm}$, (111) silicon. The inset shows selected area diffraction pattern showing the (111) plane orientation.

Thickness of the layer was of the order of $40 \mu \mathrm{m}$. The layer was opaque to $100 \mathrm{keV}$ electrons and TEM micrographs could be registered only at the edges of the layer. The micrograph shows a characteristic speckled-looking structure produced by a random network of interconnected, overlapping pores. The characteristic feature size in Fig. 3 is less than or equals about $10 \mathrm{~nm}$. Larger pores of diameter of 100-200 nm are also observed on the walls of which smaller structures develop evidencing fractal geometry of the pores. The picture shown in Fig. 3 is fairly typical of the investigated samples.

The inset in Fig. 3 shows selected area diffraction pattern (SADP) showing characteristic sixfold spot pattern of [111] family of planes in silicon. The observed spots are slightly broadened comparing to crystalline silicon but no evidence of polycrystallinity or amorphization has been found. Additional weak circles seen in SADP picture can be interpreted as resulting from the diffraction on polycrystalline material with zinc-blende structure and lattice constant $a=4.4 \AA$ which can be identified with $\beta$-SiC deposited onto the sample surface during the TEM investigation. This demonstrates that pure silicon is the dominant material composing porous layer. In particular, no traces of silicon fluorides $\mathrm{SiF}_{2}$ or $\mathrm{SiF}_{4}$ have been found in the porous material. Rapid SiC contamination, comparing to bulk material can be understood in terms of extreme reactivity of porous material, directly related to its vast surface area.

\section{Conclusion}

Based on the experimental results presented above we believe that efficient visible luminescence observed in porous silicon formed by anodic dissolution in concentrated HF electrolytes is due to the quantum size effect. However, many aspects of this luminescence are still unclear and deserve more attention in the future. 


\section{References}

[1] D.R. Turner, J. Electrochem. Soc. 105, 402 (1958).

[2] Y. Watanabe, Y. Arita, T. Yokoyama, J. Electrochem. Soc. 122, 1351 (1975).

[3] L.T. Canham, Appl. Phys. Lett. 57, 1046 (1990).

[4] K.H. Jung, S. Shih, T.Y. Hsieh, Appl. Phys. Lett. 59, 3264 (1991).

[5] C. Tsai, Appl. Phys. Lett. 59, 2814 (1991).

[6] R.P. Vasquez, Appl. Phys. Lett. 60, 1004 (1992).

[7] Z.Y. Xu, M. Gal, M. Gross, Appl. Phys. Lett. 60, 1375 (1992).

[8] H.D. Fuchs, M.S. Brandt, M. Stutzman, J. Weber, MRS Spring Meeting, San Francisco 1992, to be published. 